\title{
Graphene: The Thinnest Known Coating for Corrosion Protection
}

\author{
R.K. SINGH RAMAN ${ }^{1,2,3}$ and ABHISHEK TIWARI ${ }^{1}$ \\ 1.-Department of Mechanical and Aerospace Engineering, Monash University, Clayton, \\ VIC 3800, Australia. 2.-Department of Chemical Engineering, Monash University, Clayton, \\ VIC 3800, Australia. 3.-e-mail: raman.singh@monash.edu
}

Graphene, a two-dimensional (2-D) atomically thin film of carbon atoms, has unique characteristics that have triggered unprecedented research excitement. Besides several other unique properties, graphene possesses remarkable chemical inertness. Graphene coatings either a single or a few atomic layers thick on metals has been shown to improve their corrosion resistance by up to one-and-half orders of magnitude. Although the studies on this topic are limited to just a few carried out in 2011-2013, great variability is found in the extent of corrosion resistance from graphene reported in these studies. This article presents a brief review of graphene as a corrosion-protecting coating, identifies potential and presents some new data to suggest graphene as durable corrosion-resistant coating.

\section{NEED OF A DISRUPTIVE APPROACH TO CORROSION MITIGATION}

Corrosion of engineering alloys and its mitigation measures continue to cost dearly ( $\sim 4 \%$ of GDP of any developed economy which translates to $\sim \$ 8 \mathrm{~b}$ annually to Australia and $\sim \$ 250$ billion to USA). Traditional approaches, such as use of corrosionresistant alloys and coatings have brought about significant mitigation of the age-old problem of corrosion of metallic materials. The alloys with the highest corrosion resistance (such as duplex stainless steels, SAF 2507 and 2205) also possess very high amounts of the expensive element, chromium (22-29 wt.\%), as well as considerable amounts of another alloying element-nickel (which is even more expensive). Corrosion-resistant alloys perform on the principle of their inherent ability to develop a corrosion-resistant surface film of oxide. However, chloride ions (which are abundantly present in seawater) can disrupt this protective oxide film not only in lesser alloys but also in the case of the alloys with the highest resistance when the corrosive environment is very aggressive. Hence, the traditional mitigation strategy based on just increasing the chromium content of corrosion-resistant alloys has not proved to be long lasting, particularly in highly aggressive conditions. Therefore, the search continues for materials with very high corrosion resistance that is required for applications in which either highly corrosive fluids are handled (e.g., concentrated chloride solutions in desalination plants) or where very high corrosion resistance is required for very long durations (e.g., nuclear waste containers).

Disruptive approaches to achieve durable mitigation of corrosion are immensely attractive both for technological and commercial reasons. This manuscript reviews the novel and disruptive approach to achieving remarkable corrosion resistance resulting from ultrathin graphene coating, identifies the potential of graphene as a durable corrosion-resistant coating, and presents some new data to support this potential.

\section{ULTRATHIN GRAPHENE COATING FOR REMARKABLE CORROSION RESISTANCE}

Graphene, which is an atomically thin film of a honeycomb network of $s p^{2}$ hybridized carbon atoms (shown in Fig. $1^{1}$ ), has triggered unprecedented research excitement for its exceptional characteristics, such as its unique combination of strength and ductility. ${ }^{2}$ The 2010 Nobel Prize was awarded for graphene research. ${ }^{3}$ What is most relevant in the context of the potential application of graphene as a corrosion-resistant barrier is the remarkable 


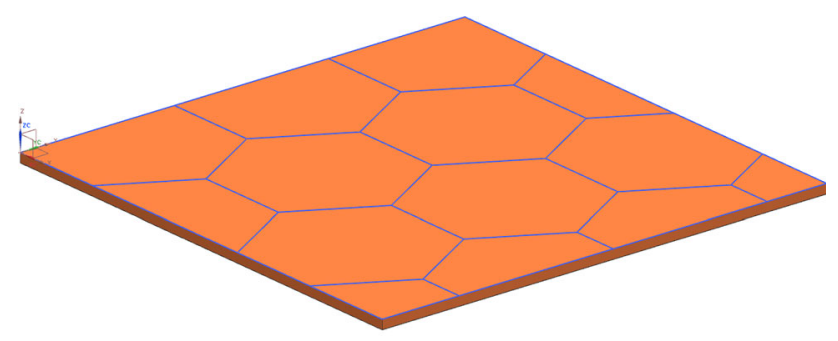

Fig. 1. Schematic diagram of a graphene sheet. ${ }^{1}$ (Reprinted with permission from Ref. 1, Copyright 2012 Australasian Corrosion Association Inc.)

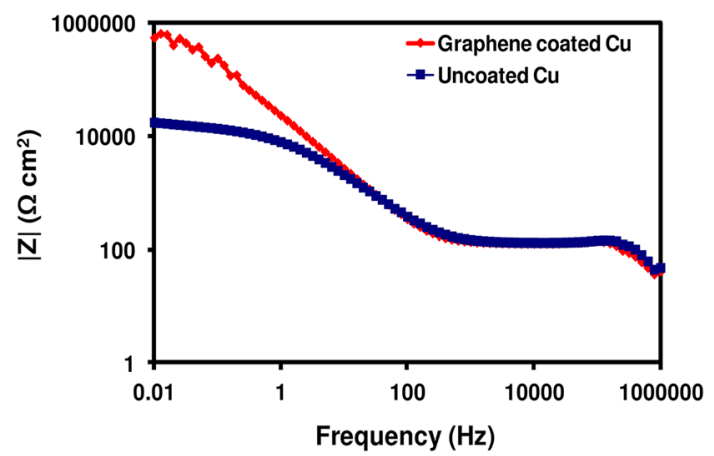

Fig. 2. Bode plots evidencing the graphene-coated $\mathrm{Cu}$ to have $\sim 2$ orders of magnitude superior corrosion resistance in seawater than the uncoated $\mathrm{Cu}^{8}$ (Note, the magnitude of $|\mathrm{Z}|$ (on the $y$ axis) at the lowest frequencies represents corrosion resistance) (Reprinted with permission from Ref. 8, Copyright 2012 Elsevier.)

chemical inertness of graphene (even to the most aggressive chemicals such as $\mathrm{HF}$ ) as well as its impermeability to fluids and gases. ${ }^{4}$ The primary requirements of an ideal surface barrier coating for corrosion resistance are its (I) inherent resistance/ immunity to degradation in aggressive environment, (II) effective resistance to permeation of corrosive fluid, and (III) mechanical integrity over the desired life of the coated components. Ceramics and carbon-based engineering materials (such as graphite) are well known to be immune to most aggressive chemicals. However, because these materials are very brittle, they suffer mechanical disruptions, and hence, have found limited use as coatings. In contrast, an atomically thin layer of graphene is reported to possess very high toughness. With its attributes of chemical inertness, toughness, and impermeability, the ultrathin graphene films possess a great potential as "the thinnest known corrosion-protecting coating." Studies hitherto, on the uses of graphene for corrosion-resistant coatings, are limited to just a few publications, ${ }^{5-9}$ and they are all very recent (published in 2011-2013). Corrosion of engineering alloys is a vexing problem, and its mitigation by traditional approaches (such as by suitable alloying

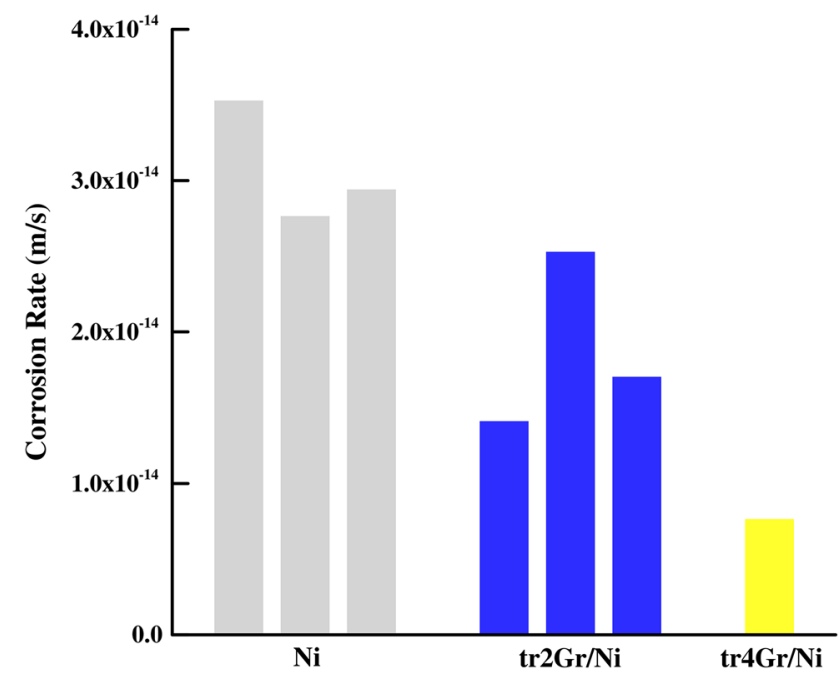

Fig. 3. Corrosion rate: $\mathrm{Gr} / \mathrm{Ni}$ is graphene coating on $\mathrm{Ni}$ by CVD, tr2 $\mathrm{Gr} / \mathrm{Ni}$ and $\operatorname{tr} 4 \mathrm{Gr} / \mathrm{Ni}$, respectively, represent mechanically transferred 2 and 4 graphene layers onto $\mathrm{Ni}$, and $\mathrm{Ni}$ is uncoated-Ni. ${ }^{5}$ (Reprinted with permission from Ref. 5, Copyright 2012 American Chemical Society.).

and traditional coating) has brought about significant but only incremental changes. In contrast, just an ultrathin (a couple of atomic layers) layer of graphene on metals has been found to bring about disruptive improvements in corrosion resistance. ${ }^{5-8}$ In their very recent work, ${ }^{8}$ the author's group has shown an ultrathin graphene coating to improve the corrosion resistance of copper by two orders of magnitude (Fig. $2^{8}$ ) in an aggressive chloride solution. This finding is the best reported improvement in corrosion resistance of copper due to graphene coating. Given the huge cost of corrosion, a durable corrosion mitigation of such alloys due to the disruptive approach of graphene coating is immensely attractive. However, a very recent study ${ }^{9}$ has the title, "Graphene as a Long-Term Metal Oxidation Barrier: Worse than Nothing"; i.e., in contrast to other studies, the graphene coating was found to drastically deteriorate the corrosion resistance of copper.

\section{ROLE OF THE NATURE OF GRAPHENE COATING IN CORROSION RESISTANCE}

The nature of graphene films profoundly influences the corrosion resistance of the graphenecoated metals. The most striking findings in this respect have come out of the work by Prasai et al. ${ }^{5}$ on the corrosion resistance of graphene-coated $\mathrm{Ni}$, produced by two different routes. The coatings developed by the chemical vapor deposition (CVD) process provided improvement by 20 times whereas those developed by mechanically transferring two or four layers of graphene onto Ni resulted in a maximum improvement by only four times (Fig. 3). ${ }^{5}$ It is extremely important to reiterate that during CVD, graphene film on $\mathrm{Ni}$ develops by the combined 


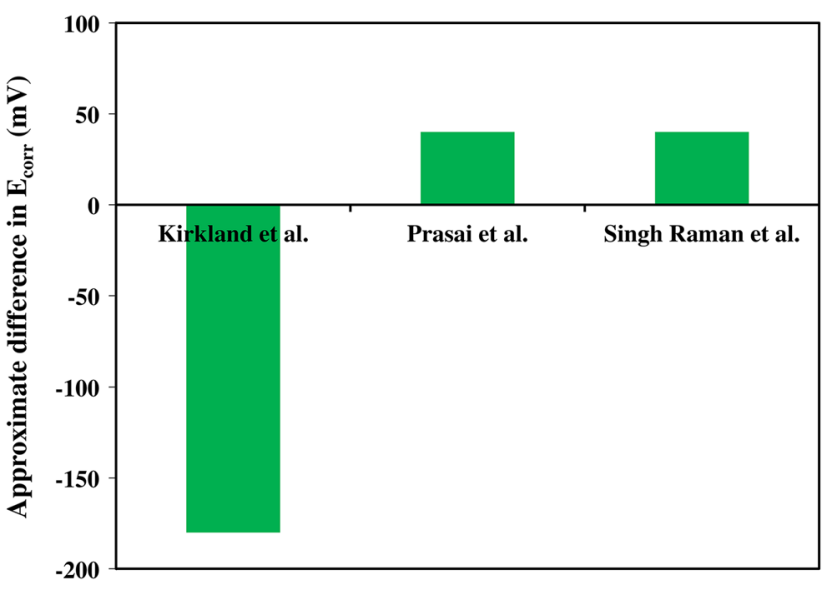

Fig. 4. Difference in the $E_{\text {corr }}$ of the graphene-coated and uncoated $\mathrm{Cu}$ as reported in the studies by Kirkland et al., ${ }^{7}$ Prasai et al., ${ }^{5}$ and Singh Raman et al. ${ }^{8}$ (Reprinted with permission from Ref. 1, Copyright 2012 Australasian Corrosion Association Inc.)

mechanism of a surface catalysis and greater solubility of carbon in $\mathrm{Ni}$ at high temperatures and the rejection of carbon from the metal matrix with cooling to allow the formation a thin surface layer of graphene. ${ }^{10,11}$ The graphene coating thus developed has a good correlation and adhesion with the substrate and, possibly, has a good surface coverage and fewer defects, which explains the superior corrosion resistance due to such graphene coating (as opposed to the coating of mechanically transferred graphene layers on Ni). ${ }^{5}$ The remarkable corrosion resistance was observed also in the commercially available graphene-coated nickel. ${ }^{7}$ But, the graphene coatings developed by mechanically transferring layers of graphene onto $\mathrm{Ni}$ apparently had channels/discontinuities that allowed ion transport and, hence, provided only up to four times improvement in corrosion resistance (Fig. 3$)^{5}$

Graphene coatings on $\mathrm{Cu}$ investigated in different studies $^{5,7,8}$ have showed different degrees of improvement in aqueous corrosion resistance (Figs. 4 and $5^{1}$ )-from little resistance ${ }^{7}$ to seven times the resistance, ${ }^{5}$ to nearly two orders of magnitude. ${ }^{8}$ In fact, in their very recently published work, Schriver et al. ${ }^{9}$ has demonstrated graphenecoated copper to show remarkably inferior oxidation resistance during long-term oxidation in air at 185$250^{\circ} \mathrm{C}$ for durations up to $17 \mathrm{~h}$. The reasons for such variations primarily arise from the quality of graphene films, i.e., irregularities/lack of complete coverage $^{7}$ and cracks in the graphene film. ${ }^{5}$

Schriver et al. ${ }^{9}$ have categorically attributed the long-term deleterious role of the coating to poor coverage at the boundaries of graphene domains and the high charge conductivity of graphene. The authors have attributed such behavior to the highly cathodic nature of carbon materials (such as graphite), which was indicated earlier by Singh Raman et al. ${ }^{8}$ in the context of corrosion resistance due to graphene.

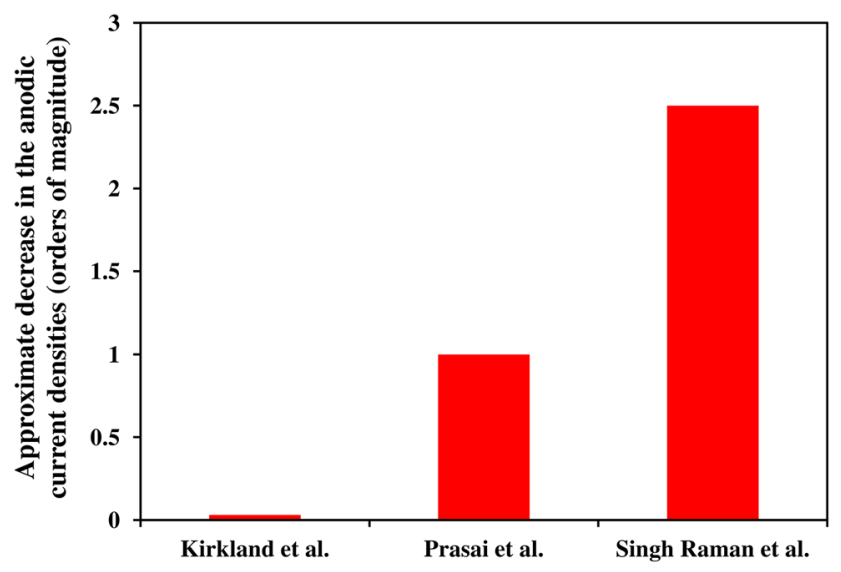

Fig. 5. Decrease in the anodic current densities of the graphenecoated and uncoated Cu reported in the studies by Kirkland et al., Prasai et al., ${ }^{5}$ and Singh Raman et al. ${ }^{8}$ (Reprinted with permission from Ref. 1, Copyright 2012 Australasian Corrosion Association Inc.)

\section{DURABLE CORROSION RESISTANCE RESULTING FROM GRAPHENE COATING}

The authors have recently investigated whether the corrosion resistance due to graphene coating that have hitherto been reported in most studies is durable. In this research, graphene was deposited on copper by chemical vapour deposition and was ascertained using Raman spectroscopy to be multilayered coating of graphene. Figure 6 presents a typical Raman spectrum of the graphene films. ${ }^{12}$ The $I_{\mathrm{G}} / I_{2-\mathrm{D}}$ ratio of 1.36 clearly indicates four or five layers of graphene coating on copper. Corrosion protection due to graphene was examined by potentiodynamic polarization and electrochemical impedance spectroscopy (EIS).

The potentiodynamic polarization of multilayer graphene-coated copper and pure copper at $1 \mathrm{~h}$ of immersion in $0.1 \mathrm{M} \mathrm{NaCl}$ suggested the anodic and cathodic current densities of multilayer graphenecoated copper to be nearly five times lower than the uncoated copper (Fig. $7^{12}$ ). Thus, the multilayer graphene coating on copper considerably decreases the dissolution of the copper. The corrosion potential $E_{\text {corr }}$ of the multilayer graphene-coated copper was $30 \mathrm{mV}$ more positive to that of the uncoated copper (Fig. $7^{12}$ ). $E_{\text {corr }}$ is the measure of corrosion susceptibility, and thus, the positive shift in $E_{\text {corr }}$ strongly indicates the corrosion protection ability of a multilayer graphene coating.

Consistent with the polarization results (Fig. $7^{12}$ ), the Bode plots of graphene-coated and uncoated samples (Fig. 8) suggest the impedance of graphene-coated copper to be nearly three times superior to that of uncoated copper. The decrease in anodic and cathodic current densities in this study is not as remarkable as shown in the study by Singh Raman et al. ${ }^{8}$ However, it is significant enough to suggest the potential of graphene as corrosion-protective coating. The lesser improvement in corrosion resis- 


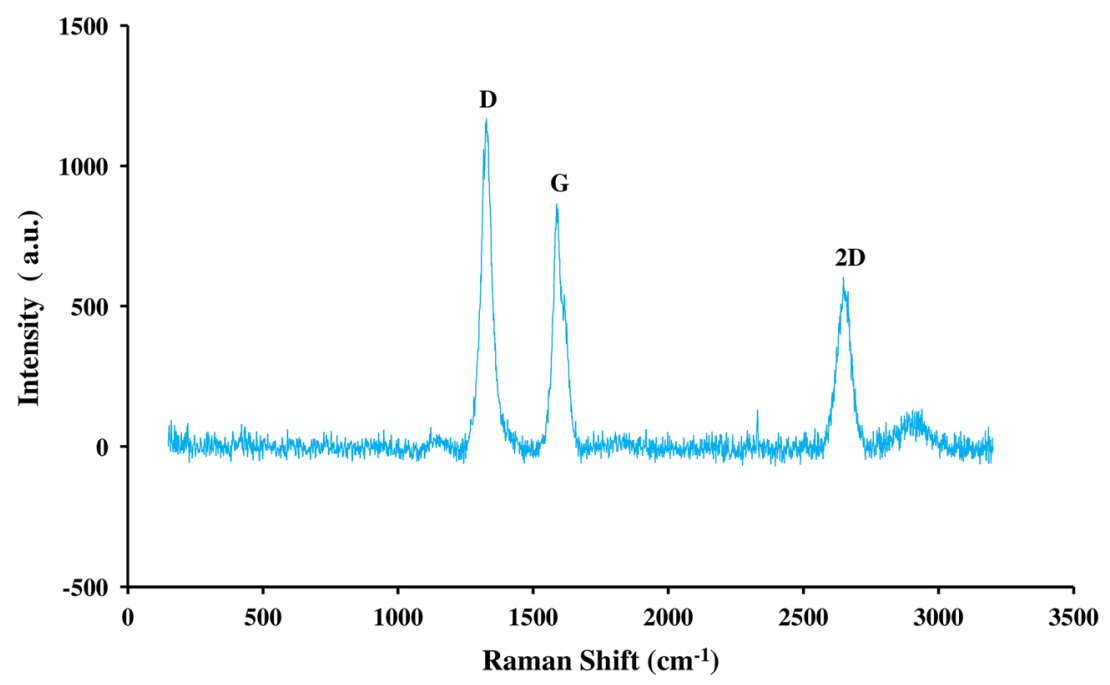

Fig. 6. Raman spectrum of graphene-coated copper sample. (Reprinted with permission from Ref. 12, Copyright 2013 Australasian Corrosion Association Inc.)

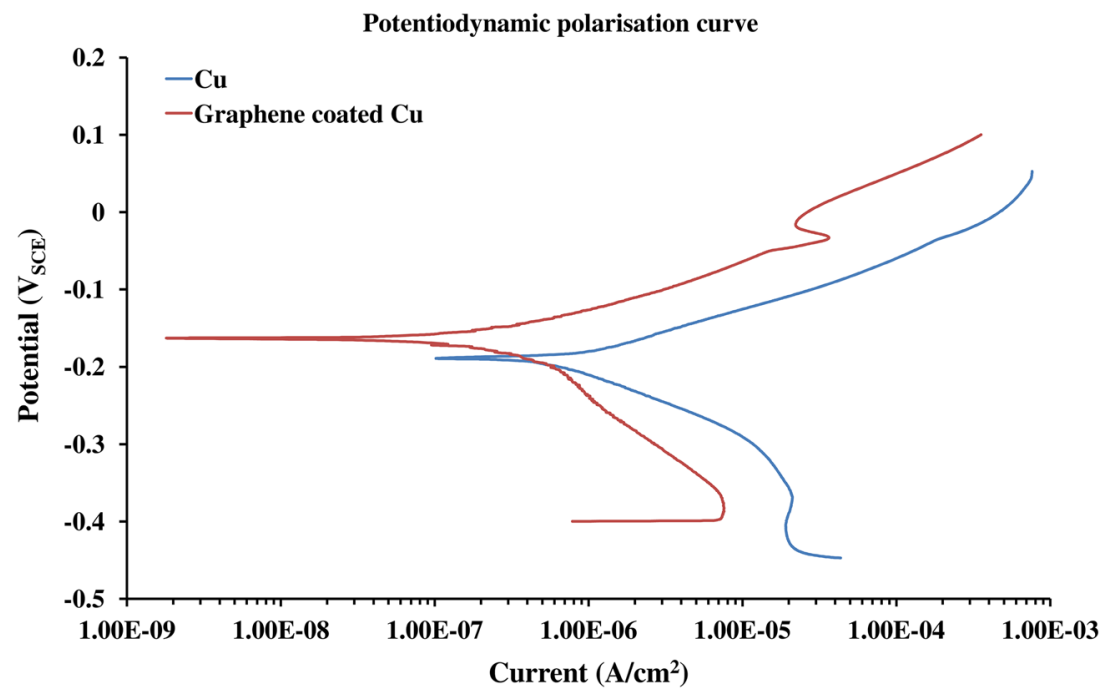

Fig. 7. Potentiodynamic polarization curve for graphene-coated and uncoated copper sample. (Reprinted with permission from Ref. 12, Copyright 2013 Australasian Corrosion Association Inc.)

tance in this study (as compared to that in study by Singh Raman et al., ${ }^{8}$ as suggested by the polarization and EIS data in Figs. 7 and $8^{12}$ and those reported in Ref. 9) can be attributed to the difference in the nature of graphene coating in the two studies. The strong D-peak (defect peak) in Fig. 6 clearly indicates that there are more conducting pathways through these defects in the coating resulting in less improvement in corrosion current densities compared to earlier monolayer or bilayer graphene. ${ }^{8}$

Although the graphene developed in the authors' recent study may not have produced as much corrosion resistance as in the earlier study, ${ }^{8}$ the most significant finding of this study is the remarkable durability of this corrosion resistance. The EIS results in Fig. 9 compare corrosion resistance of graphene-coated and uncoated copper in $0.1 \mathrm{~N} \mathrm{NaCl}$ solution. The corrosion resistance of copper remained unaltered even after a long exposure of $386 \mathrm{~h}$. This observation is the first to suggest that even a prolonged immersion in an aggressive chloride solution caused little deterioration to the corrosion resistance of the graphene-coated metal.

Contrary to our observation of improvement in corrosion resistance due to ultrathin graphene coating and those reported in the literature, a very recent study by Schriver et al. ${ }^{9}$ reported the longerterm oxidation/corrosion resistance of graphenecoated copper to be considerably inferior to uncoated copper. However, the researchers have also provided evidence of considerable defects in their graphene coating. In fact, the areas in which the 


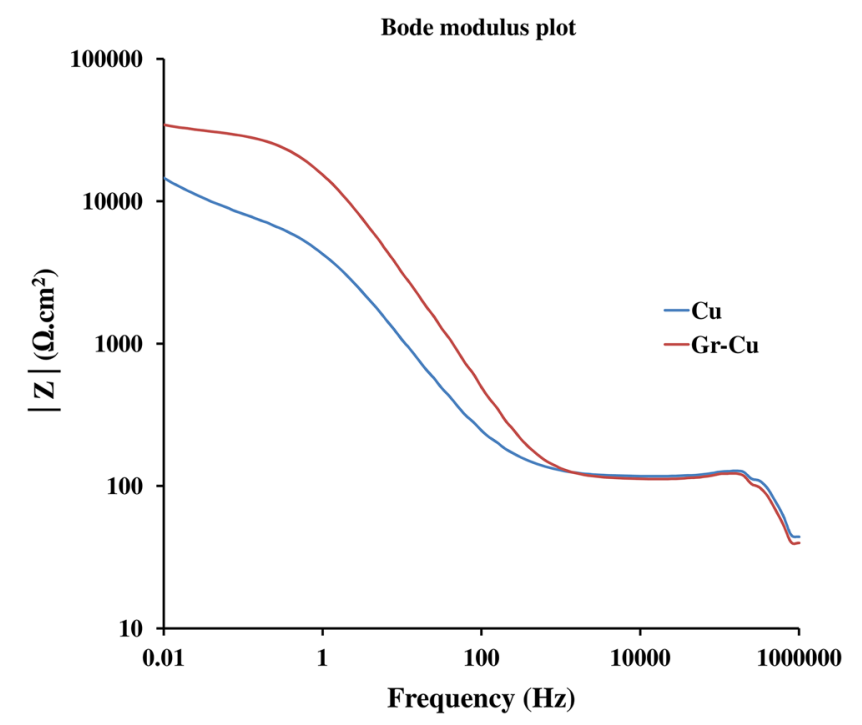

Fig. 8. Bode modulus plots for graphene-coated and uncoated copper sample. (Reprinted with permission from Ref. 12, Copyright 2013 Australasian Corrosion Association Inc.)

coating remained intact continued to show excellent oxidation/corrosion resistance over a long period. Indeed, prior to this recent report, ${ }^{9}$ Singh Raman et al. ${ }^{8}$ clearly suggested that in the event that those coatings that do not provide proper coverage on copper, the oxidation/corrosion resistance could be inferior because of the highly cathodic nature of graphene/graphite. The graphene coating that provides varying degrees of corrosion resistance and the extent of defect contents to be the cause of this variability is consistent with the comparison of studies by different groups. ${ }^{5-9}$

\section{OPPORTUNITIES IN GRAPHENE COATING FOR CORROSION RESISTANCE}

Although the reasons described above for variability in corrosion resistance due to graphene coating may be all valid, it still begs a broader question, i.e., why the graphene coating developed on $\mathrm{Cu}$ by the $\mathrm{CVD}$ process tends to produce films with such a great variability in properties. The possible reason could be that the graphene coating on $\mathrm{Cu}$ develops by a surface catalysis/adsorption mechanism, ${ }^{6,10}$ i.e., unlike in the case of CVD of graphene on $\mathrm{Ni}$ [which is governed by the combination mechanism of (I) catalysis/adsorption and (II) solubility and rejection]. The catalytic process over copper surface needs to be optimized to accomplish a homogeneous coverage.

Early methods that involved mechanically exfoliating graphene layers from graphite were suitable for small amounts and tiny area coverage. ${ }^{2}$ However, the CVD method has been employed for synthesizing larger areas of graphene on a suitable metal surface. ${ }^{13,14}$ In the CVD process for graphene deposition, a hydrocarbon is thermally decomposed into carbon atoms that arrange into graphene on

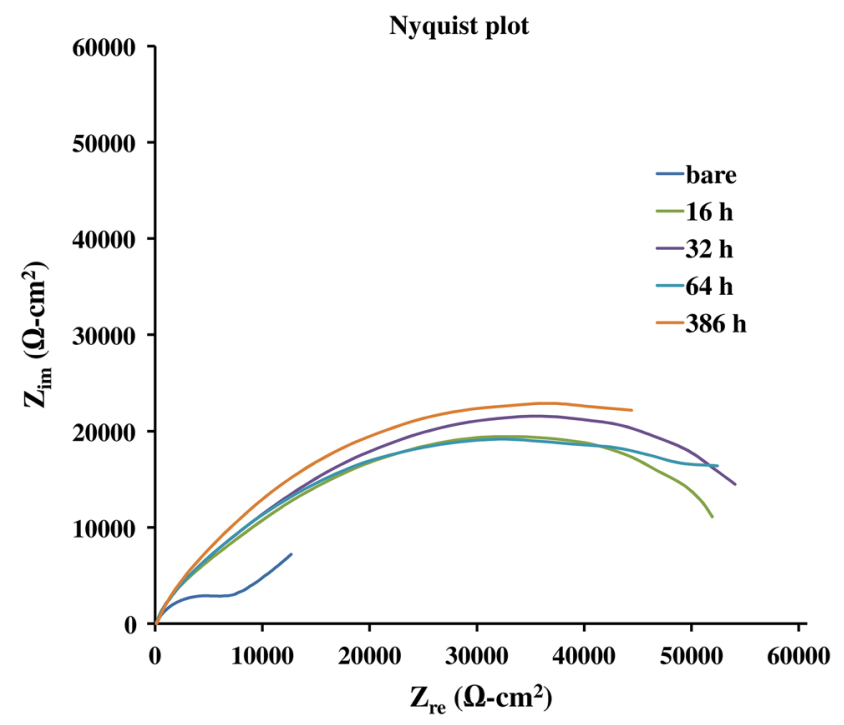

Fig. 9. Nyquist plots comparing corrosion resistance of graphenecoated and uncoated copper in $0.1 \mathrm{~N} \mathrm{NaCl}$ solution, showing the corrosion resistance of copper remains unaltered after long exposure of $386 \mathrm{~h}$.

metal substrate, as a 2-D honeycomb structure shown in Fig. 1. The methods reported so far for synthesizing CVD graphene have been successful on metals having face-centered cubic (fcc) or hexagonal close-packed (hcp) crystal structures. Hydrocarbons such as methane and ethylene have been used as precursors at decomposition temperatures of about $1000^{\circ} \mathrm{C}^{15-17}$ Metals with almost zero solubility of carbon at reaction temperature, e.g., $\mathrm{Cu}$, deposit via a catalytic mechanism and are more likely to develop a single-layer graphene instead of multilayer graphene because the catalytic effect ceases after the first layer is deposited. The metal surface acts as a catalyst, so as soon as a given site at the metal surface is covered by the depositing graphene layer, the catalytic decomposition at that site will cease, thus restricting the graphene deposition to a single layer. ${ }^{18}$ Graphene deposition on the metals having considerable solubilities of carbon at such reaction temperatures (e.g., Ni) progresses by a different and possibly complicated mechanism. In this mechanism, the carbon produced due to the hydrocarbon decomposed at the metal surface will diffuse into the bulk metal and will be rejected out during cooling because carbon solubility decreases considerably with temperature. The rate of rejection of carbon is critical to the development of a few layers of graphene. ${ }^{18}$ In this mechanism, generally a multilayer graphene will be formed instead of the single-layer graphene (that generally develops on $\mathrm{Cu}$ ). Complications can arise also when metal substrates of larger thicknesses are used. Thicker substrates will dissolve unnecessarily high amounts of carbon, which during cooling will reject too much carbon, producing graphene soot instead of a thin graphene coating. 
In employing graphene for corrosion resistance for practical applications, it is a nontrivial challenge to achieve commercial level depositions of graphene coating on common engineering alloys. However, there is a great opportunity for tailoring the deposition process for successfully developing an ultrathin graphene layer on more common engineering metals and alloys, such as mild steels, for their durable corrosion resistance.

\section{CONCLUSIONS}

Ultrathin (one or a few atomic layers thick) graphene coatings have been claimed to be "the thinnest known corrosion-protecting coating." Such coatings on copper and nickel can considerably improve their corrosion resistance (by up to 1.5 orders of magnitude) in common electrolytes. On the contrary, a very recent study has reported graphene coatings to deteriorate corrosion/oxidation resistance ("Graphene as a Long-Term Metal Oxidation Barrier: Worse than Nothing"9).

Improvement in aqueous corrosion resistance of copper due to graphene coating varies from insignificant to over 1.5 orders of magnitude, whereas the improvement for nickel (by an order of magnitude) is more consistent.

Such graphene coatings possess enormous potential as effective corrosion-resistant coatings for other engineering metals and alloys, but there is a bottleneck of successfully developing a good quality coating.

\section{REFERENCES}

1. A. Tiwari, P. Chakraborty Banerjee, R.K. Singh Raman, and M. Majumder (Paper \#133 presented at the Corrosion \& Prevention 2012 Conference, Melbourne, Australia, 2012).
2. C. Lee, X. Wei, J.W. Kysar, and J. Hone, Science 321, 385 (2008).

3. A.K. Geim and K.S. Novoselov, Nat. Mater. 6, 183 (2007).

4. E. Stolyarova, D. Stolyarov, K. Bolotin, S. Ryu, L. Liu, K.T. Rim, M. Klima, M. Hybertsen, I. Pogorelsky, I. Pavlishin, K. Kusche, J. Hone, P. Kim, H.L. Stormer, V. Yakimenko, and G. Flynn, Nano Lett. 9, 332 (2008).

5. D. Prasai, J.C. Tuberquia, R.R. Harl, G.K. Jennings, and K.I. Bolotin, ACS Nano 6, 1102 (2012).

6. S. Chen, L. Brown, M. Levendorf, W. Cai, S.-Y. Ju, J. Edgeworth, X. Li, C.W. Magnuson, A. Velamakanni, R.D. Piner, J. Kang, J. Park, and R.S. Ruoff, ACS Nano 5, 1321 (2011).

7. N.T. Kirkland, T. Schiller, N. Medhekar, and N. Birbilis, Corros. Sci. 56, 1 (2012).

8. R.K. Singh Raman, P. Chakraborty Banerjee, D.E. Lobo, H. Gullapalli, M. Sumandasa, A. Kumar, L. Choudhary, R. Tkacz, P.M. Ajayan, and M. Majumder, Carbon 50, 4040 (2012).

9. M. Schriver, W. Regan, W.J. Gannett, A.M. Zaniewski, M.F. Crommie, and A. Zettl, ACS Nano 7(7), 5763 (2013).

10. X. Li, W. Cai, L. Colombo, and R.S. Ruoff, Nano Lett. 9, 4268 (2009).

11. R.S. Weatherup, B.C. Bayer, R. Blume, C. Ducati, C. Baehtz, R. Schlögl, and S. Hofmann, Nano Lett. 11, 4154 (2011).

12. A. Tiwari and R.K. Singh Raman (Paper \#123 presented at the Corrosion \& Prevention 2013 Conference, Brisbane, Australia, 2013).

13. A. Reina, X. Jia, J. Ho, D. Nezich, H. Son, V. Bulovic, M.S. Dresselhaus, and J. Kong, Nano Lett. 9, 30 (2008).

14. A. Srivastava, C. Galande, L. Ci, L. Song, C. Rai, D. Jariwala, K.F. Kelly, and P.M. Ajayan, Chem. Mater. 22, 3457 (2010).

15. X. Li, W. Cai, J. An, S. Kim, J. Nah, D. Yang, R. Piner, A. Velamakanni, I. Jung, E. Tutuc, S.K. Banerjee, L. Colombo, and R.S. Ruoff, Science 324, 1312 (2009).

16. P.W. Sutter, J.-I. Flege, and E.A. Sutter, Nat. Mater. 7, 406 (2008).

17. J. Coraux, A.T. N'Diaye, C. Busse, and T. Michely, Nano Lett. 8, 565 (2008).

18. C. Miao, C. Zheng, O. Liang, and Y.-H. Xie, Physics and Applications of Graphene-Experiments (Rijeka, Croatia: InTech, 2011), www.intechopen.com/books/physics-and-applications-ofgraphene-experiments/chemical-vapor-deposition-of-graphene. 